KYUNGPOOK Math. J. 49(2009), 545-555

\title{
Multivalent Harmonic Uniformly Starlike Functions
}

Oм AhujA*

Department of Mathematics Kent State University, Burton, Ohio, 44021-9500, USA e-mail : oahuja@kent.edu

SANTOSH JOSHI

Department of Mathematics Walchand College of Engineering, Sangli, (M.S) India 416415

e-mail : joshisb@hotmail.com

\section{NAVENEET SANGLE}

Department of Mathematics Annasaheb Dange College of Engineering, Ashta, Sangli, (M.S) India 416301

e-mail : navneet_sangle@rediffmail.com

ABSTRACT. In this paper, we investigate a generalized family of complex-valued harmonic functions that are multivalent, sense-preserving, and are associated with $k$-uniformly harmonic functions in the unit disk. The results obtained here include a number of known and new results as their special cases.

\section{Introduction}

A harmonic function $f$ defined in a simply connected complex domain $D \subset \mathbb{C}$ can be expressed by $f(z)=h(z)+\overline{g(z)}, z \in D$. We call $h$ the analytic part and $g$ the co-analytic part of $f$. If the co-analytic part of $f$ is zero, then $f$ reduces to the analytic case. The mapping $z \rightarrow f(z)$ is sense-preserving and locally one-to-one in $D$ if and only if the Jacobian of $f$ is positive, that is, if and only if

$$
J_{f}(z)=\left|h^{\prime}(z)\right|^{2}-\left|g^{\prime}(z)\right|^{2}>0, z \in D .
$$

Denote by $H$ the family of functions $f=h+\bar{g}$ which are harmonic, sense-preserving and univalent in the open unit disk $\triangle=\{z:|z|<1\}$ with

$$
h(z)=z+\sum_{n=2}^{\infty} a_{n} z^{n}, \quad g(z)=\sum_{n=1}^{\infty} b_{n} z^{n}, \quad\left|b_{1}\right|<1 .
$$

The class $H$ was defined and studied by Clunie and Sheil-Small [10]. Also, see

* Corresponding author.

Received July 17, 2008; accepted September 12, 2008.

2000 Mathematics Subject Classification: Primary 30C45, Secondary 30C50, 30C55.

Key words and phrases: harmonic functions, multivalent harmonic functions, convex combinations, distortion bounds, uniformly harmonic starlike. 
excellent monograph entitled, 'Harmonic mapping in the plane' by Duren [11]. For a fixed positive integer $m \geq 1$, let $H(m)$ denote the family of all multivalent harmonic functions $f=h+\bar{g}$ which are sense-preserving in $\triangle$ and are of the form

$$
h(z)=z^{m}+\sum_{n=2}^{\infty} a_{n+m-1} z^{n+m-1}, \quad g(z)=\sum_{n=1}^{\infty} b_{n+m-1} z^{n+m-1},\left|b_{m}\right|<1 .
$$

Recent interest in the study of multivalent harmonic functions in the plane prompted the publication of several articles, such as [3], [4], [5], [9] and [15]. Note that $H(1) \equiv H$. We say that $f \in H(m)$ is a multivalent harmonic starlike of order $\beta, 0 \leq \beta<1$ if $f$ satisfies the condition

$$
\frac{\partial}{\partial \theta}\left(\arg \left(f\left(r e^{i \theta}\right)\right)\right) \geq m \beta
$$

for each $z=r e^{i \theta}, 0 \leq \theta<2 \pi$ and $0 \leq r<1$. Denote this class of multivalent harmonic starlike functions of order $\beta$ by $S_{H}^{*}(m, \beta)$. The classes $S_{H}^{*}(1, \beta)$ and $S_{H}^{*}(m, \beta)$ were studied in [3], [5] and [15].

Let $G_{H}(k, m, \beta, t)$ be the family of functions $f$ in $H(m)$ satisfying the inequality

$$
\operatorname{Re}\left(\frac{z f^{\prime}(z)}{z^{\prime}\left[(1-t) z^{m}+t f(z)\right]}\right) \geq k\left|\frac{z f^{\prime}(z)}{z^{\prime}\left[(1-t) z^{m}+t f(z)\right]}-m\right|+m \beta,
$$

for some $k,(0 \leq k<\infty), m(m \geq 1), \beta(0 \leq \beta<1), t(0 \leq t \leq 1), z \in \triangle$ and where

$$
z^{\prime}=\frac{\partial}{\partial \theta}\left(z=r e^{i \theta}\right), f^{\prime}(z)=\frac{\partial}{\partial \theta} f\left(r e^{i \theta}\right)=i\left(z h^{\prime}(z)-\overline{z g^{\prime}(z)}\right) .
$$

Using the fact that Rew $>k|w-m|+m \beta \Leftrightarrow \operatorname{Re}\left[\left(k e^{i \theta}+1\right) w-k m e^{i \theta}\right] \geq m \beta$, it follows from the condition (1.3) that $\mathrm{f}$ is in $G_{H}(k, m, \beta, t)$ if and only if

$$
\operatorname{Re}\left[\frac{\left(k e^{i \theta}+1\right)\left(z h^{\prime}(z)-\overline{z g^{\prime}(z)}\right)}{(1-t) z^{m}+t(h(z)+\overline{g(z)})}-k m e^{i \theta}\right] \geq m \beta .
$$

The set $G_{H}(k, m, \beta, t)$ is a comprehensive family that contains several previously studied subclasses of $H(m)$ or $H$. For example,

$$
\begin{aligned}
G_{H}(0, m, \beta, 1) & \equiv S_{H}^{*}(m, \beta) ;[3],[15] \\
G_{H}(0, m, 0,1) & \equiv S_{H}^{*}(m, 0) ;[5] \\
G_{H}(0,1, \beta, 1) & \equiv S_{H}^{*}(1, \beta) \equiv S_{H}^{*}(\beta) ;[16] \\
G_{H}(0,1,0,1) & \equiv S_{H}^{*}(0) \equiv S_{H}^{*} ;[24],[25]
\end{aligned}
$$




$$
\begin{aligned}
G_{H}(0, m, \beta, 0) & \equiv R_{H}(m, \beta):=\left\{f \in H(m): \operatorname{Re}\left(\frac{f^{\prime}(z)}{\frac{\partial}{\partial \theta}\left(z^{m}\right)}\right) \geq m \beta, 0 \leq \beta<1\right\} ;[4] \\
G_{H}(0,1, \beta, 0) & \equiv R_{H}(1, \beta) \equiv R_{H}(\beta) ;[2] \\
G_{H}(1, m, \beta, 1) & \equiv G_{H}(m, \beta):=\left\{f \in H(m): \operatorname{Re}\left(\left(1+e^{i \alpha}\right) \frac{z f^{\prime}(z)}{z^{\prime} f(z)}-m e^{i \alpha}\right) \geq m \beta\right\} ; \\
G_{H}(1,1, \beta, 1) & \equiv G_{H}(1, \beta) \equiv G_{H}(\beta) ;[23] \\
G_{H}(k, 1, \beta, t) & \equiv G_{H}(k, \beta, t) .[1]
\end{aligned}
$$

Let $S(m)$ be the well known family of functions $h$ in $H(m)$ that are analytic and univalent in $\triangle$ and are of the form $h(z)=z^{m}+\sum_{n=2}^{\infty} a_{n+m-1} z^{n+m-1}, z \in \triangle$. We observe that $S(1) \subset S, S(m) \subset H(m)$, and $G_{s}(k, m, \beta, t) \subset G_{H}(k, m, \beta, t)$. Also the family $G_{s}(k, m, \beta, t)$ contains several previously studied subclasses of analytic functions in $\triangle$. For example

$$
\begin{aligned}
G_{s}(0,1, \beta, t) & :=\left\{h \in S: \operatorname{Re} \frac{z h^{\prime}(z)}{(1-t) z+t h(z)}>\beta\right\} ;[7],[20] \\
G_{s}(1,1, \beta, 1) & :=\left\{h \in S: \operatorname{Re}\left(\frac{z h^{\prime}(z)}{h(z)}\right) \geq\left|\frac{z h^{\prime}(z)}{h(z)}-1\right|+\beta\right\} ;[8] \\
G_{s}(k, 1,0,1) & \equiv k-S T:=\left\{h \in S: \operatorname{Re}\left(\frac{z h^{\prime}(z)}{h(z)}\right) \geq k\left|\frac{z h^{\prime}(z)}{h(z)}-1\right|\right\} ;[18] \\
G_{s}(1,1,0,1) & \equiv U S T \equiv 1-S T ;[19],[21],[22] .
\end{aligned}
$$

Finally, we define the family

$$
G_{\bar{H}}(k, m, \beta, t):=T H(m) \cap G_{H}(k, m, \beta, t),
$$

where $T H(m), m \geq 1$ denote the class of functions $f=h+\bar{g}$ in $H(m)$ so that $h$ and $g$ are of the form

$$
h(z)=z^{m}-\sum_{n=2}^{\infty}\left|a_{n+m-1}\right| z^{n+m-1}, \quad g(z)=\sum_{n=1}^{\infty}\left|b_{n+m-1}\right| z^{n+m-1}, z \in \triangle .
$$

The class $T H(m)$ was first studied in [5].

In this paper, we investigate coefficient conditions, extreme points, and distortion bounds for functions in the families $G_{H}(k, m, \beta, t)$ and $G_{\bar{H}}(k, m, \beta, t), m \geq 1$. We also examine their convolution and convex combination properties. We remark that the results so obtained for these general families can be viewed as extensions and generalizations for various subclasses of $S, H, S(m)$, and $H(m)$ as listed previously in this section. 


\section{Main results}

We first prove sufficient coefficient conditions for harmonic functions in $G_{H}(k, m, \beta, t)$. These conditions are shown to be necessary for the functions in $G_{\bar{H}}(k, m, \beta, t)$.

Theorem 1. Let $f=h+\bar{g}$ be so that $h$ and $g$ are given by (1.2). If

$$
\begin{aligned}
& \sum_{n=2}^{\infty} \frac{(n+m-1)(k+1)-t m(k+\beta)}{m(1-\beta)+1-|m(1-\beta)-1|}\left|a_{n+m-1}\right| \\
& +\sum_{n=1}^{\infty} \frac{(n+m-1)(k+1)+t m(k+\beta)}{m(1-\beta)+1-|m(1-\beta)-1|}\left|b_{n+m-1}\right| \leq \frac{1}{2},
\end{aligned}
$$

when $k \geq 0, m \geq 1,0 \leq \beta<1$ and $0 \leq t \leq 1$, then $f \in G_{H}(k, m, \beta, t)$.

Proof. Suppose that $(2.1)$ holds. It suffices to prove that $\operatorname{Re}\{A(z) / B(z)\}>0$, where

$$
\begin{aligned}
& A(z)=\left(k e^{i \theta}+1\right)\left(z h^{\prime}(z)-\overline{z g^{\prime}(z)}\right)-m\left(k e^{i \theta}+\beta\right)\left((1-t) z^{m}+t h(z)+\overline{\operatorname{tg}(z)}\right), \\
& B(z)=(1-t) z^{m}+t h(z)+\overline{\operatorname{tg}(z)} .
\end{aligned}
$$

Using the fact that Rew $\geq 0$ if and only if $|1+\omega| \geq|1-\omega|$ it suffices to show that

$$
|A(z)+B(z)|-|A(z)-B(z)| \geq 0 .
$$

Substituting for $A(z)$ and $B(z)$ in (2.2), we obtain

$$
\begin{aligned}
& |A(z)+B(z)|-|A(z)-B(z)| \\
= & \mid(m(1-\beta)+1) z^{m} \\
& +\sum_{n=2}^{\infty}\left[((n+m-1)-m \beta t+t)+k e^{i \theta}((n+m-1)-m t)\right] a_{n+m-1} z^{n+m-1} \\
& -\sum_{n=1}^{\infty}\left[((n+m-1)+m \beta t-t)+k e^{i \theta}((n+m-1)+m t)\right] \bar{b}_{n+m-1}(\bar{z})^{n+m-1} \mid \\
& -\mid(m(1-\beta)-1) z^{m} \\
& -\sum_{n=2}^{\infty}\left[((n+m-1)-m \beta t-t)+k e^{i \theta}((n+m-1)-m t)\right] a_{n+m-1} z^{n+m-1} \\
& -\sum_{n=1}^{\infty}\left[((n+m-1)+m \beta t+t)+k e^{i \theta}((n+m-1)+m t)\right] \bar{b}_{n+m-1}(\bar{z})^{n+m-1} \mid
\end{aligned}
$$




$$
\begin{aligned}
\geq & (m(1-\beta)+1-|m(1-\beta)-1|)|z|^{m} \\
& \times\left\{1-\sum_{n=2}^{\infty} \frac{2[(n+m-1)(k+1)-t m(k+\beta)]}{m(1-\beta)+1-|m(1-\beta)-1|}\left|a_{n+m-1}\right|\left|z^{n-1}\right|\right. \\
& \left.-\sum_{n=1}^{\infty} \frac{2[(n+m-1)(k+1)+t m(k+\beta)]}{m(1-\beta)+1-|m(1-\beta)-1|}\left|b_{n+m-1}\right|\left|z^{n-1}\right|\right\} \\
\geq \quad & (m(1-\beta)+1-|m(1-\beta)-1|)|z|^{m} \\
& \times\left\{1-\sum_{n=2}^{\infty} \frac{2[(n+m-1)(k+1)-t m(k+\beta)]}{m(1-\beta)+1-|m(1-\beta)-1|}\left|a_{n+m-1}\right|\right. \\
& \left.-\sum_{n=1}^{\infty} \frac{2[(n+m-1)(k+1)+t m(k+\beta)]}{m(1-\beta)+1-|m(1-\beta)-1|}\left|b_{n+m-1}\right|\right\} .
\end{aligned}
$$

This last expression is non-negative by the hypothesis and so the proof is complete. The functions

$$
\begin{aligned}
f(z)=z^{m} & +\sum_{n=2}^{\infty} \frac{m(1-\beta)+1-|m(1-\beta)-1|}{2[(n+m-1)(k+1)-t m(k+\beta)]} x_{n+m-1} z^{n+m-1} \\
& +\sum_{n=1}^{\infty} \frac{m(1-\beta)+1-|m(1-\beta)-1|}{2[(n+m-1)(k+1)+t m(k+\beta)]} \bar{y}_{n+m-1}(\bar{z})^{n+m-1}
\end{aligned}
$$

where $\sum_{n=2}^{\infty}\left|x_{n+m-1}\right|+\sum_{n=1}^{\infty}\left|y_{n+m-1}\right|=1$, show that the coefficient bound given by (2.1) is sharp.

Corollary 1. Let $f=h+\bar{g}$ be so that $h$ and $g$ are given by (1.2). Also, let $m \geq 1 /(1-\beta), 0 \leq \beta<1$ and $0 \leq t \leq 1$. If the condition

$$
\begin{aligned}
& \sum_{n=2}^{\infty}[(n+m-1)(k+1)-t m(k+\beta)]\left|a_{n+m-1}\right| \\
& +\sum_{n=1}^{\infty}[(n+m-1)(k+1)+t m(k+\beta)]\left|b_{n+m-1}\right| \leq 1
\end{aligned}
$$

is satisfied, then $f \in G_{H}(k, m, \beta, t)$.

Corollary 2. Let $f=h+\bar{g}$ be so that $h$ and $g$ are given by (1.2). Also, suppose $1 \leq m \leq 1 /(1-\beta), 0 \leq \beta<1$ and $0 \leq t \leq 1$. If the condition

$$
\begin{aligned}
& \sum_{n=2}^{\infty}[(n+m-1)(k+1)-t m(k+\beta)]\left|a_{n+m-1}\right| \\
& +\sum_{n=1}^{\infty}[(n+m-1)(k+1)+t m(k+\beta)]\left|b_{n+m-1}\right| \leq m(1-\beta)
\end{aligned}
$$


holds, then $f \in G_{H}(k, m, \beta, t)$.

Theorem 2. Let $f=h+\bar{g}$ be so that $h$ and $g$ are given by (1.5). Also, let $k \geq 0,0 \leq t \leq 1$ and $0 \leq \beta<1$. Furthermore,

(i) if $1 \leq m \leq 1 /(1-\beta)$, then $f \in G_{\bar{H}}(k, m, \beta, t)$ if and only if

$$
\begin{aligned}
& \sum_{n=2}^{\infty}[(n+m-1)(k+1)-t m(k+\beta)]\left|a_{n+m-1}\right| \\
& +\sum_{n=1}^{\infty}[(n+m-1)(k+1)+t m(k+\beta)]\left|b_{n+m-1}\right| \leq m(1-\beta) ;
\end{aligned}
$$

(ii) if $m(1-\beta) \geq 1$, then $f \in G_{\bar{H}}(k, m, \beta, t)$ if and only if

$$
\begin{aligned}
& \sum_{n=2}^{\infty}[(n+m-1)(k+1)-t m(k+\beta)]\left|a_{n+m-1}\right| \\
& +\sum_{n=1}^{\infty}[(n+m-1)(k+1)+t m(k+\beta)]\left|b_{n+m-1}\right| \leq 1 .
\end{aligned}
$$

Proof. In view of Corollary 1 and Corollary 2, it suffices to show that $f \in$ $G_{\bar{H}}(k, m, \beta, t)$ if the condition (2.4) does not hold. We note that a necessary and sufficient condition for $f=h+\bar{g}$, given by (1.5), to be in $G_{\bar{H}}(k, m, \beta, t)$ is that the coefficient condition (1.4) to be satisfied. Equivalently, we must have

$$
\operatorname{Re}\left\{\frac{\left(k e^{i \theta}+1\right)\left(z h^{\prime}(z)-\overline{z g^{\prime}(z)}\right)-m\left(k e^{i \theta}+\beta\right)\left((1-t) z^{m}+t h(z)+\overline{t g(z)}\right)}{(1-t) z^{m}+t h(z)+\overline{t g(z)}}\right\} \geq 0 .
$$

Upon choosing the value of $z$ on the positive real axis and using $\operatorname{Re}\left(-e^{i \theta}\right) \geq-\left|e^{i \theta}\right|=$ -1 , where $0 \leq|z|=r<1$, the above inequality reduces to

$$
\begin{aligned}
& \left\{m(1-\beta)-\sum_{n=2}^{\infty}((n+m-1)(k+1)-m t(k+\beta))\left|a_{n+m-1}\right| r^{n-1}\right. \\
& \left.-\sum_{n=1}^{\infty}((n+m-1)(k+1)+m t(k+\beta))\left|b_{n+m-1}\right| r^{n-1}\right\} \\
& \times\left\{1-\sum_{n=2}^{\infty}\left|a_{n+m-1}\right| r^{n-1}+t \sum_{n=1}^{\infty}\left|b_{n+m-1}\right| r^{n-1}\right\}^{-1} \geq 0 .
\end{aligned}
$$

If condition (2.5) does not hold then the numerator of (2.6) is negative for $r$ sufficiently close to 1 because of conditions (i) or (ii). Thus there exits $z_{0}=r_{0}>1$, for which the left side of (2.6) is negative. This contradicts the required condition for $f \in G_{\bar{H}}(k, m, \beta, t)$. Using definition (1.3), and according to the arguments given in [2] and [16], we obtain distortion bounds for the functions in $G_{\bar{H}}(k, m, \beta, t)$ in 
Theorem 3 and extreme points of the closed convex hulls of $G_{\bar{H}}(k, m, \beta, t)$, denoted by $\operatorname{clco} G_{\bar{H}}(k, m, \beta, t)$, in Theorem 4 . The proofs of Theorems 3 and 4 are similar to the corresponding results in [2] and [16] and so are omitted.

Theorem 3. If $f \in G_{\bar{H}}(k, m, \beta, t)$, then for $|z|=r<1$

$$
\begin{aligned}
& |f(z)| \\
& \leq\left\{\begin{array}{r}
\left(1+\left|b_{m}\right|\right) r^{m}+\left(\frac{m(1-\beta)}{(m+1)(k+1)-t m(k+\beta)}-\frac{m(k+1)+t m(k+\beta)}{(m+1)(k+1)-t m(k+\beta)}\left|b_{m}\right|\right) r^{m+1}, \\
\text { if } m(1-\beta) \leq 1 \\
\left(1+\left|b_{m}\right|\right) r^{m}+\left(\frac{1}{(m+1)(k+1)-t m(k+\beta)}-\frac{m(k+1)+t m(k+\beta)}{(m+1)(k+1)-t m(k+\beta)}\left|b_{m}\right|\right) r^{m+1}, \\
\text { if } m(1-\beta) \geq 1
\end{array}\right.
\end{aligned}
$$

$$
\begin{aligned}
& |f(z)| \\
& \geq\left\{\begin{array}{r}
\left(1-\left|b_{m}\right|\right) r^{m}-\left(\frac{m(1-\beta)}{(m+1)(k+1)-t m(k+\beta)}-\frac{m(k+1)+t m(k+\beta)}{(m+1)(k+1)-t m(k+\beta)}\left|b_{m}\right|\right) r^{m+1}, \\
\text { if } m(1-\beta) \leq 1 \\
\left(1-\left|b_{m}\right|\right) r^{m}-\left(\frac{1}{(m+1)(k+1)-t m(k+\beta)}-\frac{m(k+1)+t m(k+\beta)}{(m+1)(k+1)-t m(k+\beta)}\left|b_{m}\right|\right) r^{m+1}, \\
\text { if } m(1-\beta) \geq 1
\end{array}\right.
\end{aligned}
$$

Corollary 3. If $f \in G_{\bar{H}}(k, m, \beta, t)$, then

$$
\left\{\omega:|\omega|<\left[\begin{array}{r}
1-\frac{m(1-\beta)}{(m+1)(k+1)-t m(k+\beta)}-\frac{(k+1)-2 t m(k+\beta)}{(m+1)(k+1)-t m(k+\beta)}\left|b_{m}\right| \text { if } m(1-\beta) \leq 1 \\
1-\frac{(k+1)-2 t m(k+\beta)}{(m+1)(k+1)-t m(k+\beta)}-\frac{1}{(m+1)(k+1)-t m(k+\beta)}\left|b_{m}\right| \text { if } m(1-\beta) \geq 1
\end{array}\right]\right\}
$$

Theorem 4. A function $f$ is in $c l c o G_{\bar{H}}(k, m, \beta, t)$ if and only if it can be expressed in the form

$$
f(z)=\sum_{n=1}^{\infty}\left(X_{n+m-1} h_{n+m-1}+Y_{n+m-1} g_{n+m-1}\right)
$$

where

$$
h_{m}(z)=z^{m},
$$

$$
\begin{aligned}
& h_{n+m-1}(z) \\
& = \begin{cases}z^{m}-\frac{m(1-\beta)}{(n+m-1)(k+1)-t m(k+\beta)} z^{n+m-1}(n=2,3,4, \cdots), & \text { if } m(1-\beta) \leq 1 \\
z^{m}-\frac{1}{(n+m-1)(k+1)-t m(k+\beta)} z^{n+m-1}(n=2,3,4, \cdots) & \text { if } m(1-\beta) \geq 1\end{cases}
\end{aligned}
$$


$g_{n+m-1}(z)$
$= \begin{cases}z^{m}+\frac{m(1-\beta)}{(n+m-1)(k+1)+t m(k+\beta)}(\bar{z})^{n+m-1}(n=1,2,3, \cdots), & \text { if } m(1-\beta) \leq 1 \\ z^{m}+\frac{1}{(n+m-1)(k+1)+t m(k+\beta)}(\bar{z})^{n+m-1}(n=1,2,3, \cdots), & \text { if } m(1-\beta) \geq 1\end{cases}$

and

$$
\sum_{n=1}^{\infty}\left(X_{n+m-1}+Y_{n+m-1}\right)=1, X_{n+m-1} \geq 0 \text { and } Y_{n+m-1} \geq 0 .
$$

In particular, the extreme points of $G_{\bar{H}}(k, m, \beta, t)$ are $\left\{h_{n+m-1}\right\}$ and $\left\{g_{n+m-1}\right\}$.

In the next two theorems, we prove that the class $G_{\bar{H}}(k, m, \beta, t)$ is invariant under convolution and convex combinations of its members. We first recall that for harmonic functions

$$
f(z)=z^{m}-\sum_{n=2}^{\infty}\left|a_{n+m-1}\right| z^{n+m-1}+\sum_{n=1}^{\infty}\left|b_{n+m-1}\right|(\bar{z})^{n+m-1}
$$

and

$$
F(z)=z^{m}-\sum_{n=2}^{\infty}\left|A_{n+m-1}\right| z^{n+m-1}+\sum_{n=1}^{\infty}\left|B_{n+m-1}\right|(\bar{z})^{n+m-1}
$$

in $T H(m)$, the convolution of $f$ and $F$ is defined as

$$
\begin{aligned}
& (f * F)(z)=f(z) * F(z) \\
= & z^{m}-\sum_{n=2}^{\infty}\left|a_{n+m-1} A_{n+m-1}\right| z^{n+m-1}+\sum_{n=1}^{\infty}\left|b_{n+m-1} B_{n+m-1}\right|(\bar{z})^{n+m-1} .
\end{aligned}
$$

Using this definition, we first show that $G_{\bar{H}}(k, m, \beta, t)$ is closed under convolution.

Theorem 5. For $0 \leq \alpha \leq \beta<1$, let $f \in G_{\bar{H}}(k, m, \beta, t)$ and $F \in G_{\bar{H}}(k, m, \alpha, t)$, then

$$
f * F \in G_{\bar{H}}(k, m, \beta, t) \subset G_{\bar{H}}(k, m, \alpha, t) .
$$

Proof. Let, $f, F \in G_{\bar{H}}(k, m, \beta, t)$ be given by (2.7) and (2.8), respectively. Note that the coefficients of $f$ and $F$ must satisfy the conditions similar to the inequality (2.4). For $F \in G_{\bar{H}}(k, m, \alpha, t)$ we observe that $\left|A_{n+m-1}\right| \leq 1$ and $\left|B_{n+m-1}\right| \leq 1$. 
Since

$$
\begin{aligned}
& \quad \sum_{n=2}^{\infty} \frac{(n+m-1)(k+1)-t m(k+\beta)}{m(1-\beta)+1-|m(1-\beta)-1|}\left|a_{n+m-1}\right|\left|A_{n+m-1}\right| \\
& \quad+\sum_{n=1}^{\infty} \frac{(n+m-1)(k+1)+t m(k+\beta)}{m(1-\beta)+1-|m(1-\beta)-1|}\left|b_{n+m-1}\right|\left|B_{n+m-1}\right| \\
& \leq \quad \sum_{n=2}^{\infty} \frac{(n+m-1)(k+1)-t m(k+\beta)}{m(1-\beta)+1-|m(1-\beta)-1|}\left|a_{n+m-1}\right| \\
& \quad+\sum_{n=1}^{\infty} \frac{(n+m-1)(k+1)+t m(k+\beta)}{m(1-\beta)+1-|m(1-\beta)-1|}\left|b_{n+m-1}\right| .
\end{aligned}
$$

The right hand side of the above inequality is bounded by 1 because $f \in$ $G_{\bar{H}}(k, m, \beta, t)$. Therefore the result follows.

Finally, we determine the convex combination of the members of $G_{\bar{H}}(k, m, \beta, t)$.

Theorem 6. The class $G_{\bar{H}}(k, m, \beta, t)$ is closed under convex combination.

Proof. For $i=1,2,3, \cdots$ suppose $f_{i} \in G_{\bar{H}}(k, m, \beta, t)$, where $f_{i}$ are given by

$$
f_{i}(z)=z^{m}-\sum_{n=2}^{\infty}\left|a_{i_{n+m-1}}\right| z^{n+m-1}+\sum_{n=1}^{\infty}\left|b_{i_{n+m-1}}\right|(\bar{z})^{n+m-1}
$$

For $\sum_{i=1}^{\infty} t_{i}=1,0 \leq t_{i} \leq 1$, the convex combination of $f_{i}$ may be written as

$$
\sum_{i=1}^{\infty} t_{i} f_{i}(z)=z^{m}-\sum_{n=2}^{\infty}\left(\sum_{i=1}^{\infty} t_{i}\left|a_{i_{n+m-1}}\right|\right) z^{n+m-1}+\sum_{n=1}^{\infty}\left(\sum_{i=1}^{\infty} t_{i}\left|b_{i_{n+m-1}}\right|\right)(\bar{z})^{n+m-1} .
$$

Since

$$
\begin{aligned}
& \sum_{n=2}^{\infty}[(n+m-1)(k+1)-\operatorname{tm}(k+\beta)]\left|a_{i_{n+m-1}}\right| \\
& +\sum_{n=1}^{\infty}[(n+m-1)(k+1)+t m(k+\beta)]\left|b_{i_{n+m-1}}\right| \\
& \leq\left\{\begin{array}{lll}
m(1-\beta), & \text { if } & m(1-\beta) \geq 1, \\
1, & \text { if } & m(1-\beta) \leq 1,
\end{array}\right.
\end{aligned}
$$

it follows from the above equation 


$$
\begin{aligned}
\sum_{n=2}^{\infty}[(n+m-1) & (k+1)-\operatorname{tm}(k+\beta)] \sum_{i=1}^{\infty} t_{i}\left|a_{i_{n+m-1}}\right| \\
& +\sum_{n=1}^{\infty}[(n+m-1)(k+1)+t m(k+\beta)] \sum_{i=1}^{\infty} t_{i}\left|b_{i_{n+m-1}}\right| \\
=\sum_{i=1}^{\infty} t_{i}\left\{\sum_{n=2}^{\infty}[(n+m-1)(k+1)-t m(k+\beta)]\left|a_{i_{n+m-1}}\right|\right. & \\
+ & \left.\sum_{n=1}^{\infty}[(n+m-1)(k+1)+t m(k+\beta)]\left|b_{i_{n+m-1}}\right|\right\} \\
& \leq\left\{\begin{array}{lll}
m(1-\beta) \sum_{i=1}^{\infty} t_{i}=m(1-\beta), & \text { if } \quad m(1-\beta) \leq 1, \\
\sum_{i=1}^{\infty} t_{i}=1, & \text { if } \quad m(1-\beta) \geq 1,
\end{array}\right.
\end{aligned}
$$

and so $\sum_{i=1}^{\infty} t_{i} f_{i}(z) \in G_{\bar{H}}(k, m, \beta, t)$.

\section{References}

[1] O. P. Ahuja, R. Aghalary and S. B. Joshi, Harmonic univalent functions associated with $k$-uniformly starlike functions, Math. Sci. Res. J., 9(1)(2005), 9-17.

[2] O. P. Ahuja and J. M. Jahangiri, Noshiro-type harmonic univalent functions, Scientiae Math. Japonicae, 56(2)(2002), 293-299.

[3] O. P. Ahuja and J. M. Jahangiri, Multivalent harmonic starlike functions with missing coefficients, Math. Sci. Res. J., 7(9)(2003), 347-352.

[4] O. P. Ahuja and J. M. Jahangiri, On a linear combination of classes of multivalently harmonic functions, Kyungpook Math. J., 42(1)(2002), 61-70.

[5] O. P. Ahuja and J. M. Jahangiri, Multivalent harmonic starlike functions, Ann. Univ. Mariae Curie-Sklodowska, Vol. LV, 1 Sectio A, 55(1)(2001), 1-13.

[6] O. P. Ahuja and J. M. Jahangiri, Errata to "Multivalent harmonic starlike functions" [Univ. Mariae Curie-Sklodowska, Vol LV, sectio A, 55(1)(2001), 1-13], Univ. Mariae Curie-Sklodowska, Sectio. A, 56(1)(2002), 105.

[7] M. K. Aouf, A generalization of multivalent functions with negative coefficient II, Bull Korean Math. Soc., 25(2)(1988), 221-232.

[8] R. Bharati, R. Parvatham and A. Swaminathan, On subclasses of uniformly convex functions and corresponding class of starlike functions, Tamkang J. Math., 25(1997), $17-32$. 
[9] D. Bshouty, W. Hengartner and M. Naghibi-Beidokhti, p-valent harmonic mapping with finite Blaschke dilatations, Ann. Univ. Mariae Curie. Sklodowska Sectio. A, 53(2)(1999), 9-26.

[10] J. Clunie and T. Sheil-Small, Harmonic univalent functions, Ann. Acd. Aci. Fenn. Ser. A I Math., 9(1984), 3-25.

[11] P. L. Duren, Harmonic mappings in the plane, Cambridge Tract in Mathematics (Cambridge: 156, Cambridge University Press), 2004, ISBN: 0-521-64121-73.

[12] P. L. Duren, W. Hengartner and R. S. Laugesen, The argument principles harmonic functions, Amer. Math. Monthly, 103(5)(1996), 411-415.

[13] A. W. Goodman, On uniformly convex functions, Ann. Polon, Math., 56(1)(1991), 87-92.

[14] A. W. Goodman, On uniformly starlike functions, J. Math. Anal. Appl., 155(1991), 364-370.

[15] H. O. Guney and O. P. Ahuja, Inequalities involving multipliers for multivalent harmonic functions, J. Ineq. Pure Appl. Math., 7(5), Article 190, (2006), 1-9.

[16] J. M. Jahangiri, Harmonic functions starlike in the unit disc, J. Math. Anal. Appl., 235(1999), 470-477.

[17] J. M. Jahangiri, G. Murugusundaramoorthy and K. Vijaya, On starlikeness of certain multivalent Harmonic functions, Journal of Natural Geometry, 24(2003), 1-10.

[18] S. Kanas and H. M. Srivastava, Linear operators associated with k-uniformly convex functions, Integral Transform. Spect. Funct., 9(2)(2000), 121-132.

[19] W. Ma and D. Minda, Uniformly convex functions, Ann Polon Math., 57(1992), 165-175.

[20] J. Patel, On a class of p-valent functions with negative and missing coefficients, Kyungpook Math. J., 36(1996), 29-40.

[21] F. Ronning, On uniform starlikeness and related properties of univalent functions, Complex variables Theory Appl., 24(1994), 233-239.

[22] F. Ronning, Uniformly convex functions and a corresponding class of starlike functions, Proc. Amer. Math. Soc., 118(1993), 189-196.

[23] T. Rosy, B. A, Stephen, K. G. Subramanian and J. M. Jahangiri, Goodman-RonningType harmonic univalent functions, Kyungpook Math. J., 41(2001), 45-54.

[24] H. Silverman, Harmonic univalent functions with negative coefficients, J. Math. Anal. Appl., 220(1998), 283-289.

[25] H. Silverman and E. M. Silvia, Subclasses of harmonic univalent functions, New Zealand J. Math., 28(1999), 275-284. 